\title{
Validation of the self-completed Cambridge-Hopkins questionnaire (CH-RLSq) for ascertainment of restless legs syndrome (RLS) in a population survey
}

\author{
Richard P. Allen $^{\mathrm{a}, *}$, Brendan J. Burchell ${ }^{\mathrm{b}}$, Ben MacDonald ${ }^{\mathrm{c}}$, Wayne A. Hening ${ }^{\mathrm{d}}$, Christopher J. Earley ${ }^{\mathrm{a}}$ \\ a Department of Neurology, Johns Hopkins University, Asthma and Allergy Building, 1B76B, 5501 Hopkins Bayview Circle, Baltimore, MD 21224, United States \\ ${ }^{\mathrm{b}}$ Faculty of Social and Political Sciences, University of Cambridge, Cambridge, UK \\ ${ }^{\mathrm{c}}$ Statistical Consultant, Cambridge, UK \\ ${ }^{\mathrm{d}}$ Department of Neurology, UMDNJ-Robert Wood Johnson Medical School, New Brunswick, NJ, United States
}

\section{A R T I C L E I N F O}

\section{Article history:}

Received 22 March 2008

Received in revised form 23 October 2008 Accepted 30 October 2008

Available online $\mathrm{xxxx}$

\section{Keywords:}

Restless legs

RLS

Restless legs syndrome

RLS diagnosis

Questionnaire validation

RLS diagnostic questionnaire

Hopkins telephone diagnostic interview

Cambridge-Hopkins RLS questionnaire (CHRLSq)

\begin{abstract}
A B S T R A C T
Background and purpose: Epidemiological studies of restless legs syndrome (RLS) have been limited by lack of a well validated patient-completed diagnostic questionnaire that has a high enough specificity to provide a reasonable positive predictive value. Most of the currently used patient completed diagnostic questionnaires have neither been validated nor included items facilitating the differential diagnosis of RLS from conditions producing similar symptoms. The Cambridge-Hopkins diagnostic questionnaire for RLS (CH-RLSq) was developed with several iterations to include items covering the basic diagnostic features of RLS and to provide some basic differential diagnosis. This validation study sought to determine the sensitivity and specificity of the RLS diagnosis based on this questionnaire.

Patients and methods: The CH-RLSq was completed by 2005 blood donors who were asked to consent to being contacted for a telephone diagnostic interview. A scoring criterion was established for ascertainment of RLS based on the clinical definition of the disorder and the exclusion of "mimic" conditions. A weighted sample $(N=185)$ of all completed questionnaires was selected for expert clinical diagnosis of RLS using the validated Hopkins Telephone Diagnostic Interview (HDTI). The telephone interviewers were blinded to all questionnaire responses.

Results: A telephone diagnosis was obtained on 183 of the sample's 185 questionnaires. The questionnaire's normalized sensitivity and specificity were $87.2 \%$ and $94.4 \%$, respectively, for RLS compared to not RLS. The positive predictive values in this sample were $85.5 \%$.

Conclusions: The Cambridge-Hopkins RLS questionnaire provides a reasonable level of sensitivity and specificity for ascertainment of RLS in population-based studies.
\end{abstract}

(c) 2008 Elsevier B.V. All rights reserved.

\section{Introduction}

The diagnosis of restless legs syndrome (RLS) relies upon clinical history indicating presence of all four of the essential diagnostic criteria: an urge to move the legs when at rest that is worse in the evening or night and relieved by movement [1]. Abnormally frequent periodic limb movements when resting (PLMW) or asleep (PLMS) provide an objective sign of the disorder that is sensitive (occurs in at least $80 \%$ of RLS patients [2]) but not specific enough (PLMS/ $h>15$ occurs in $40 \%$ of adults over 65 [3]) to be useful for diagnosis. RLS occurs commonly (about 5-10\% in European and North American populations $[4,5]$ ), but can have a wide range of severity from intermittent, to only occurring when provoked by prolonged rest late in the day, to persistent and easily provoked whenever rest occurs [1]. Despite the now well-defined diagnostic criteria, the wide range of severity and lack of a specific objective sign complicate

\footnotetext{
* Corresponding author. Tel.: +1 410550 2609; fax: +1 4105503364

E-mail address: richardjhu@mac.com (R.P. Allen).
}

accurate diagnosis. Moreover, the differential diagnosis includes several common conditions that produce RLS-like symptoms, i.e., leg-muscle cramps, positional discomfort, anxious fidgeting, and focal leg pains. Most of these conditions that mimic RLS symptoms can be easily identified in a clinical situation, but are not so easy to exclude through a subject-completed questionnaire.

The large-scale epidemiological studies needed to uncover population and environmental factors contributing to RLS require a reasonably accurate subject-completed diagnostic instrument. The four essential diagnostic criteria have provided the basis for most questionnaires used in epidemiological studies. These essential criteria were intended for use in a clinical setting and not as the basis for a subject-completed questionnaire. The criteria are deceptively simple and sometimes even in the clinical setting can lead to hasty diagnosis without careful consideration of differential diagnosis. Questions covering only the 4 criteria are particularly insufficient for a reasonably valid subject-completed RLS ascertainment questionnaire. Epidemiological studies that have relied upon some questionnaire formulations of these four diagnostic questions have rarely 
checked their ascertainment validity against a clinical diagnosis of RLS.

Ascertainment questionnaires can be evaluated in terms of their sensitivity, specificity and positive predictive value relative to a clinically accepted diagnostic standard. Sensitivity is defined as the proportion of ill patients who are correctly diagnosed, i.e., the number diagnosed divided by the total number with the disease. Specificity is the proportion of unaffected individuals who are not diagnosed with the disease. Both sensitivity and specificity are defined to be independent of the proportion of the population who has the disease. The positive predictive value of a test is the proportion of those identified by a test as having the disease who do actually have the disease. Unlike sensitivity and specificity, the positive predictive value is influenced by the prevalence of the disease in the population, decreasing with decreasing prevalence [6]. While the positive predictive value is critical for studies attempting to either characterize features of a disorder or to define morbidity or comorbid conditions, it is rarely reported or even discussed.

This is particularly a problem for RLS since even a reasonably high diagnostic sensitivity of $90 \%$ and specificity of $85 \%$ for a disorder with a prevalence of $10 \%$ produces a positive predictive value of only $40 \%$, meaning 6 out of 10 subjects who are categorized as ill by the test would be falsely identified as having the disorder. Significantly improving positive predictive value for RLS requires increasing specificity above $90 \%$ without significant loss of sensitivity. A recent study compared diagnoses by a trained physician clinical interview with that from a questionnaire diagnosis covering only the four diagnostic criteria without attention to differential diagnoses. The questionnaire had a positive predictive value of only $57.9 \%$ [7]. Recognizing this problem we set out to develop a questionnaire with very high specificity (i.e., good at correctly identifying well subjects) and acceptable sensitivity (good at correctly identifying subjects with the disease) in order to achieve a more optimal positive predictive value. We recognized that any effort to increase specificity is likely to reduce sensitivity, but, as noted for most epidemiological studies, very high specificity is essential.

\section{Methods}

\subsection{Questionnaire development}

Our efforts to develop a better questionnaire started with a set of 10 questions developed by 3 RLS experts. These covered not only the four diagnostic criteria but also questions about: focal nature of the experience, complaint of restless feeling, degree feeling could not be resisted, circadian pattern in the past, RLS frequency and complaint of pain. The wording of the questions was reviewed by the experts and by a small convenience group including 10 RLS patients and their unaffected spouses or close friends. Based on patient feedback, some of the questions were reworded slightly, e.g., the question on relief with movement was reworded to emphasize the relief occurred while moving and not after movement stopped. The questionnaire containing these items was then used in a small study evaluating agreement with a subsequent clinician diagnosis for 48 out patients in a sleep disorders clinic that included an emphasis on treating RLS [8]. The questions were again reviewed for wording confusion reported by the patients, and after slight rewording were used in a larger study based in a family practice clinic in a small town in the northwestern USA. This clinic had been selected in part because prior screening items had suggested a high prevalence of RLS [9]. In this second study those diagnosed with RLS by the questionnaire and a random sample of those not diagnosed were further evaluated for RLS by a primary care doctor who had been trained in making the diagnosis. The questionnaire had $93 \%$ sensitivity and $84 \%$ specificity in this general medical setting [10]. A review of diagnostic errors indicated that the low specificity was due in part to the questionnaire failing to adequately exclude those with leg cramps and positional discomfort. The circadian pattern question also failed to adequately cover the afternoon as well as evening and night as a time for more pronounced symptoms with reduced symptoms in the morning. Questions were added to cover these items and a set of items were added to characterize significant features of RLS including frequency of symptoms, age of onset, family history, occurrence of involuntary movements and history of symptom change. A category of "probable" RLS was also established which involved patients reporting that $90 \%$ or more of the time RLS occurred while sitting, not while lying down. This gave a final 22-item questionnaire with 7 of the items used to make the diagnosis and the remaining to further characterize the condition. The respondents' answers to the final version of the CH-RLSq permitted classification as "RLS" or "not RLS." The first two questions in the final version were used to introduce to the respondents the sensations that are associated with RLS, and the remaining diagnostic questions were given in the first part of the CH-RLSq. The content of the critical questions and scoring for the RLS diagnoses is provided in Table 1 . The full questionnaire along with a recommended 13-item short-form (CH-RLSq13) of the diagnostic questionnaire covering only diagnosis (8 items in Table 1 ) and critical severity and phenotype determinations ( 5 items) can be obtained from the corresponding author.

\subsection{Validation study}

The questionnaire was used in a large population-based study of RLS among 2005 blood donors in England, a setting somewhat enriched with those reporting RLS symptoms. This population is described in detail in a companion article in Sleep Medicine [11]. From the population of blood donors, a sample of 185 questionnaires was selected for a subsequent separate telephone-administered clinical interview. Since most of the questionnaires would be from those with no RLS symptoms, an adequate validation required a stratified sample of questionnaires enriched with both RLS positive cases and borderline cases (i.e., those positive on all but one or two of the critical diagnostic questions on the questionnaire). In order to calculate coefficients that were unbiased for the entire sample of 2005 cases, the data were weighted within categories defined by the number of RLS symptoms they reported; this adjusted for the increased probability of selection of positive and borderline cases. Checks on the data verified that the weighted sample of 185 cases gave a distribution of RLS diagnoses identical to the sample of 2005 cases.

All those who completed the CH-RLSq questionnaire were asked if they would have a telephone interview and $74.6 \%$ of the subjects consented to this follow-up. This telephone interview was conducted by one of two qualified RLS experts and authors of this paper (RA, WH) using the validated structured Hopkins telephone diagnostic interview (HTDI) $[12,13]$. All telephone interviews conducted were blinded to the information on the diagnostic questionnaire and were conducted within 2-4 months after the questionnaire had been completed by the subject. The HTDI includes writing down a subject's responses on an interview form. The HTDI interview forms were then reviewed by the other expert blinded to the diagnosis previously made; when the two experts disagreed the case was discussed to reach a consensus diagnosis. In very few cases the subject was telephoned again to attempt to clarify some specific information. The first two questions of both the questionnaire and the HTDI ask if the subject had any abnormal feeling or an urge to move their legs when resting. Two of the sub- 
Table 1

Critical diagnostic questions from the CH-RLSq.

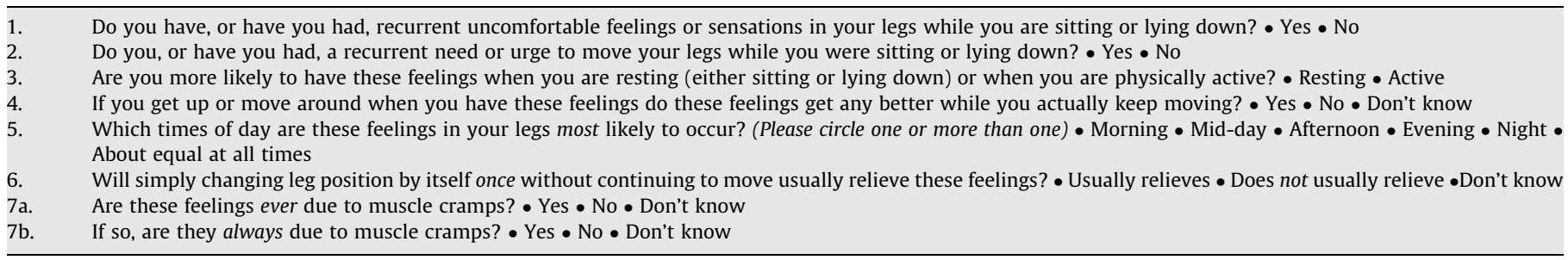

\section{Scoring:}

Definite RLS: 1 yes, 2 yes, 3 resting, 4 yes, 5 NOT equal or morning, 6 does not usually relieve, 7 a as No OR b as No.

jects denied this on the questionnaire, but when telephoned they indicated that their condition now included these feelings; since this represented a self-reported change in status their data were not included, leaving a total of 183 subjects.

\subsection{Statistical analyses}

The sensitivity, specificity and positive predictive value of the questionnaire diagnoses compared to the HTDI were calculated for the weighted data applicable to the entire sample.

\section{Results}

The questionnaire compared to clinicians' diagnoses of RLS diagnosis had a sensitivity of $87.2 \%(48 / 55)$ and specificity of $94.4 \%$ (120/127), giving a positive predictive value of $87.2 \%$ (48/ 55 ) for this particular sample of blood donors (See Table 2).

\section{Discussion}

The CH-RLSq is the first validated self-completed RLS ascertainment questionnaire and the only one that includes questions designed to exclude common conditions that produce symptoms similar to those of RLS. It is also the only one validated in a general population, that of UK blood donors, and not in a clinical patient population. Our goal was to maximize specificity by adding questions to facilitate differential diagnosis, thereby discriminating RLS from common conditions known to produce similar symptoms. Our intentions were that the questions for differential diagnosis would not cause an appreciable loss of sensitivity and would therefore improve the positive predictive value of the questionnaire, particularly in situations in populations where RLS is less common. We largely achieved our goal since the questionnaire had a specificity of $94.4 \%$. The sensitivity, as expected, decreased modestly compared to that observed with a smaller set of diagnostic questions in the study done in a primary care practice [10]. But that decrease may also reflect the greater medical, social and educational diversity of the blood donors compared to patients in a small town primary care office in Northwestern United States. In general the

Table 2

Weighted number of cases by questionnaire and HTDI diagnosis for RLS defined by combining definite and probable RLS.

\begin{tabular}{llll}
\hline Questionnaire ascertainment & $\begin{array}{l}\text { HTDI (Hopkins telephone } \\
\text { diagnostic Interview). } \\
\end{array}$ & Total questionnaire \\
\cline { 2 - 3 } & RLS & Not RLS & \\
\hline RLS & 47 & 7 & 54 \\
Not RLS & 7 & 121 & 128 \\
Total HTDI & 54 & 128 & 182 \\
\hline
\end{tabular}

(The numbers in each of the cells have been calculated from the weighted data and rounded to the nearest whole number. For this table this rounding produces numbers that add to one less than the number in the sample evaluated.) sensitivity and specificity of this questionnaire would be expected to be even better in a clinical setting than in the volunteer and generally healthy blood donor population. The positive predictive value, of course, improves in populations enriched with RLS patients.

The current study includes two sampling biases. First, although a poster was displayed at the post-donation rest area, we have no information about the number of donors who did not complete the questionnaire. This problem was further complicated by the human subjects' requirements that on the poster participants must be informed it was a study about restless legs syndrome. This likely led to increased probability of participation by donors with leg symptoms, creating a bias for higher prevalence. This bias was neither a problem for the primary goals of that study relating RLS to frequency of donation nor to the determination of sensitivity and specificity of the CH-RLSq, but it would increase the positive predictive value. A second sample bias occurs because approximately $25 \%$ of those completing the diagnostic questionnaire did not consent to a telephone interview. There was no indication this would alter the evaluation of accuracy of the questionnaire. The blood donor population also probably differs somewhat from the general population in that they include more from an educated middleclass willing to volunteer. A less-educated population might introduce more errors related to failure to carefully read or understand the questions. The questions, however, had been selected to be direct, easily understood with minimal conditional clauses, and in the process of developing the questionnaire they were reviewed by subjects from a wide range of educational backgrounds. Nonetheless, further studies in other populations would be needed to determine how much these results can be generalized.

The positive predictive value of $87.2 \%$ was acceptable for this blood-donor population. In a population with a lower prevalence of RLS, the positive predictive value will decrease. In particular, this questionnaire, when used in a population with an overall RLS prevalence of $10 \%$, would be expected to have a positive predictive value of $63.4 \%$. As noted above, the prior version of the questionnaire did not include items designed to exclude "mimics," and when evaluated in a primary care medical practice it gave sensitivity of $93 \%$ and specificity of $84 \%$ [10], yielding a positive predictive for a $10 \%$ RLS prevalence of about only $39.2 \%$. Thus, the current questionnaire provides considerable improvement over the positive predictive value of the prior versions that used only questions focused on the basic diagnostic criteria ignoring differential diagnosis. The validity of epidemiological studies that ascertain potential risk factors for RLS or determine its relative morbidity depends upon a robust positive predictive value, and accordingly, the critical factor is specificity of the diagnostic instrument. Situations where less than $60 \%$ of the subjects identified as having RLS may actually have the disorder reduce confidence in any postulated factors associated with RLS. Thus future work on questionnaire diagnosis for RLS should emphasize improving specificity in order to improve positive predictive value. One area, for example, not well covered in the current questionnaire is that related to situations 
that provoke symptoms. Adding questions covering this feature of the disorder may further enhance specificity.

Any future use of this questionnaire in languages other than English will, of course, require the appropriate linguistic validation process. Ideally any use of the questionnaire in a different population should include a validation of the questionnaire in that population. Overall this version of the Cambridge-Hopkins diagnostic questionnaire provides for the first-time a well-validated patientcompleted questionnaire for ascertainment of subjects likely to be diagnosed with RLS. It has adequate sensitivity and specificity for most epidemiological surveys.

\section{Acknowledgements}

This research was supported in part by NIH grant P01 AG021190 and by an unrestricted grant from GlaxoSmithKline. The authors would also like to thank Dr. E. Caffrey for access to the Blood Donors, the staff of the blood donor sessions for their assistance in administering the questionnaire, and Jessica Miller for fieldwork management and data collection.

\section{References}

[1] Allen RP, Picchietti D, Hening WA, Trenkwalder C, Walters AS, Montplaisir J. Restless legs syndrome: diagnostic criteria, special considerations, and epidemiology. A report from the restless legs syndrome diagnosis and epidemiology workshop at the National Institutes of Health. Sleep Med 2003;4(2):101-19.
[2] Montplaisir J, Boucher S, Poirier G, Lavigne G, Lapierre O, Lesperance P. Clinical, polysomnographic, and genetic characteristics of restless legs syndrome: a study of 133 patients diagnosed with new standard criteria. Mov Disord 1997;12(1):61-5.

[3] Ancoli-Israel S, Kripke DF, Klauber MR, Mason WJ, Fell R, Kaplan O. Periodic limb movements in sleep in community dwelling elderly. Sleep 1991;14:496-500.

[4] Berger K, Luedemann J, Trenkwalder C, John U, Kessler C. Sex and the risk of restless legs syndrome in the general population. Arch Intern Med 2004;164(2):196-202.

[5] Allen RP, Walters AS, Montplaisir J, Hening W, Myers A, Bell TJ, et al. Restless legs syndrome prevalence and impact: REST general population study. Arch Intern Med 2005;165(11):1286-92.

[6] Altman DG, Bland JM. Diagnostic tests 2: predictive values. BM] 1994;309(6947):102.

[7] Allen RP, Stillman P, Myers AJ. Patients with clinically relevant RLS in European primary care experience significant sleep disruption. Sleep Med 2007;8(Suppl. 1):S93.

[8] Allen R, Earley C. Validation of a diagnostic questionnaire for the restless legs syndrome (RLS). Neurology 2001;58(8 Suppl. 3):A4.

[9] Nichols DA, Allen RP, Grauke JH, Brown JB, Rice ML, Hyde PR, et al. Restless legs syndrome symptoms in primary care: a prevalence study. Arch Intern Med 2003;163(19):2323-9.

[10] Nichols DA, Kushida CA, Allen RP, Grauke JH, Brown JB, Rice ML, et al. Validation of RLS diagnostic questions in a primary care practice. Sleep 2003;26:A346.

[11] Burchell BJ, Allen RP, Miller JK, Hening WA, Earley CJ. RLS and Blood Donation. Sleep Med 2009. doi:10.1016/i.sleep.2008.09.013..

[12] Hening W, Allen RP, Thanner S, Washburn T, Heckler D, Walters A, et al. The Johns Hopkins telephone diagnostic interview for the restless legs syndrome: preliminary investigation for validation in a multi-center patient and control population. Sleep Med 2003;4(2):137-41.

[13] Hening WA, Allen RP, Washburn M, Lesage S, Earley CJ. Validation of the Hopkins telephone diagnostic interview for restless legs syndrome. Sleep Med 2008;9(3):283-9. 